\title{
Application of On-line HPLC-CD Detection in Separation and Structure Elucidation of Tricyclic Cephalosporine Oxaanalogues with Chromane Moiety
}

\section{Magdalena Woźnica and Jadwiga Frelek*}

Institute of Organic Chemistry, Polish Academy of Sciences, Kasprzaka 44/52, 01-224 Warsaw, Poland

In recent years, compounds containing in skeleton chromane moiety have gained the huge interest of medicinal chemists. Chromane and its derivatives are important intermediates and interesting building-blocks in organic synthesis and drug design. Beyond the most well-known derivatives of vitamin $\mathrm{E}$ with antioxidant properties [1], flavones, 3-benzylidene, 4-chromanone, or chiral dihydrocoumarin derivatives have attracted much attention due to their pharmacological and physiological activities such as anti-inflammatory, HIV replication inhibition and antioxidant $[2,3]$. A group of phenylalanine and 2 -chromanone derivatives was found to be the $\beta$-lactamase substrates [4]. Inhibition of those enzymes plays the crucial role in the discovery and development process of new active $\beta$-lactam antibacterial agents [5]. Together with the isolation from nature or synthetic studies of new active compounds their chemical analysis calls for access to modernized methods for in-depth structural characterization. The boundaries in this field lie in an improvement of detection sensitivity of small quantities, separation of complex mixtures of compounds, and in the elucidation of the stereostructure of components [6]. The assignment of absolute configuration (AC) of organic molecules still remains as one of the most important challenges in modern organic chemistry.

In this Editorial, we will focus on the application of on-line HPLCCD detection supported by TD-DFT calculation in separation and structure elucidation of $\beta$-lactam analogues possessing chromane chromophore (Figure 1, compound 1,2 ). The combination of two structural fragments, i.e., $\beta$-lactam ring and chromane, both exhibiting a broad biological activity, makes presented tricyclic cephalosporin derivatives the vast field of interest not only for systematic chiroptical studies but also biological. Our preliminary results of the biological activity of some representatives of this group of compounds have shown that compounds 1 and $\mathbf{2}$ presented in Figure 1 act as DDpeptidase 64-575 inhibitors with $\mathrm{IC}_{50}$ at $\mathrm{mmol} / \mathrm{L}$ level [7] suggesting that further structural modifications may bring new valuable antibacterial substances.

Compounds $\mathbf{1}$ and $\mathbf{3}$ were obtained in a form of optically pure enantiomers from commercially available Kaneka azetidinone [(2R,3R)-3-((1R )-1-[ tert -butyl(dimethyl)sililoxy]ethyl)-4-oxo2-azetydinone]. Readily available 4-formyloxyazetidinone was enantioselectively transformed into enantiomerically enriched (i.e. 48\%) 3,4-benzo- 2-hydroxy-5-oxacephams 2 upon treatment with 0.1 equiv. of the cinchona alkaloid in toluene [8]. Racemic 4- chromanol 4 is commertially available.

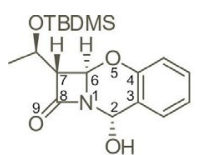

1

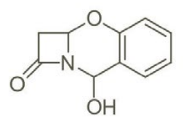

2

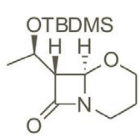

3

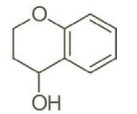

4
Figure 1: Chemical structure of compounds under study.
In fact, presented tricyclic $\beta$-lactam analogues are composed of two chromophores namely: $\beta$-lactam 3 and 4 -chromanol 4 . Therefore, in the ECD spectra of $\mathbf{1}$ and $\mathbf{2}$ contributions from both chromophores should be visible. In accordance with the $\beta$-lactam helicity rule, previously established for bicyclic analogues of penicillin and cephalosporin, the positive sign of the Cotton effect at around $220 \mathrm{~nm}$ corresponds to the negative sign of the $\mathrm{O}(9)=\mathrm{C}(8)-\mathrm{N}(1)-\mathrm{C}(2)$ torsion angle of the amide moiety and $6 R$ absolute configuration [9-11]. Primary observation has shown that compounds 1 follow the rule. However, its ECD spectrum (Figure 2, green curve) substantially has differed from ECD spectrum of the classic analogue 3 (Figure 2, red curve) and additionally has shown greater similarity to the spectrum of $4 R$-chromanol (Figure 2, navy blue curve). In light of this, the validity of the helicity rule for tricyclic cephalosporine oxaanalogues could not have been unambiguously defining. The above statement was formulated based only on the experimental results, and theoretical confirmation was required. Therefore, the theoretical TD-DFT experiments were conducted to determine electronic structure of single chromophores $\mathbf{3}$ and $\mathbf{4}$ followed by theoretical consideration of arbitrarily chosen enantiomer of $\mathbf{2}$.

The TD-DFT analysis of 4-chromanol was preceded by the chiral HPLC separation of its racemic mixture combined with the on-line recording of ECD spectra of eluted enantiomers (Figure 3A). Utilization of on-line CD/HPLC system has allowed for the fast chiral analysis of studied enantiomers. ECD spectra of optically pure compounds

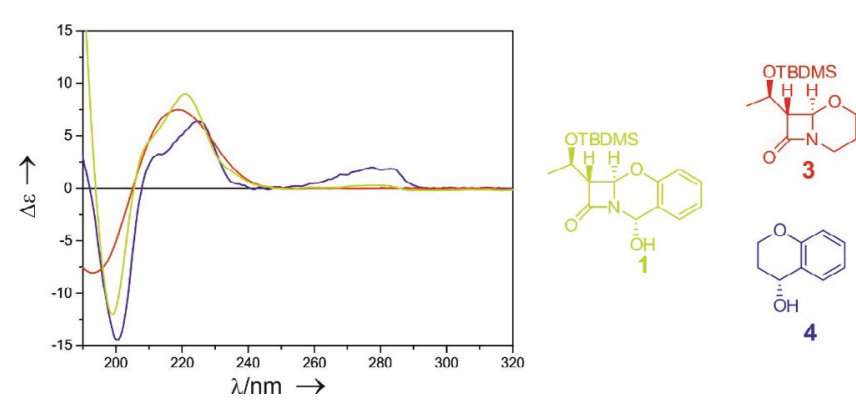

Figure 2: ECD spectra for optically pure compounds 1, 3 and 4 recorded in acetonitrile.

*Corresponding author: Jadwiga Frelek, Institute of Organic Chemistry, Polish Academy of Sciences, Kasprzaka 44 / 52, 01 - 224 Warsaw, Poland, Tel: +48 22 3432215; Fax: +48 22 6328861; E-mail: jadwiga.frelek@icho.edu.pl

Received April 19, 2016; Accepted April 20, 2016; Published April 23, 2016

Citation: Woźnica M, Frelek J (2016) Application of On-line HPLC-CD Detection in Separation and Structure Elucidation of Tricyclic Cephalosporine Oxaanalogues with Chromane Moiety. J Bioanal Biomed 8: e136. doi:10.4172/1948 593X.1000e136

Copyright: (c) 2016 Woźnica M, et al. This is an open-access article distributed under the terms of the Creative Commons Attribution License, which permits unrestricted use, distribution, and reproduction in any medium, provided the original author and source are credited. 
could have been recorded without previous tedious separation and finally, compared with the results of the calculation. Simulation of ECD spectrum of $4 R$-chromanol was performed on B3LYP/TZVP level using Gaussian 09 package [12]. The theoretical spectrum of $4 R$-chromanol stayed in a very good agreement with the spectrum of enantiomer which was eluted as a second peak $\left(t_{r}=8.9\right.$ min, Figure $3 \mathrm{~B})$ and therefore this peak was assigned to $4 R$ enantiomer whereas the first one $\left(t_{r}=7.8 \mathrm{~min}\right)$ to the $4 S$ enantiomer of chromanol. Both, experimental and theoretical ECD spectra of $4 R$-chromanol proved the presence of two positive Cotton effects in the range of diagnostic band of helicity rule (at around $220 \mathrm{~nm}$ ).

TD-DFT calculations (B3LYP/TZVP) of chiroptical properties of compounds 3 showed that the diagnostic for the helicity rule Cotton effect at around $220 \mathrm{~nm}$ is induced by the single excitation (Ex. 1) which can be described as a $n \rightarrow \pi^{*}$ amide transition (Figure 4).

In the case of enantiomerically enriched mixture of 2 (i.e $48 \%$ ) the procedure of ECD/HPLC methodology was applied. At HPLC condition same as for 4-chromanol enantiomers of $\mathbf{2}$ were separated and their ECD spectra were recorded. By the comparison of calculated ECD spectrum and experimental ones the absolute configuration could have been assigned for each eluted enantiomer. According to the TDDFT calculations in the diagnostic range for the helicity rule (at around $220 \mathrm{~nm}$ ) in the ECD spectrum of compound $6 R-2$ there were three

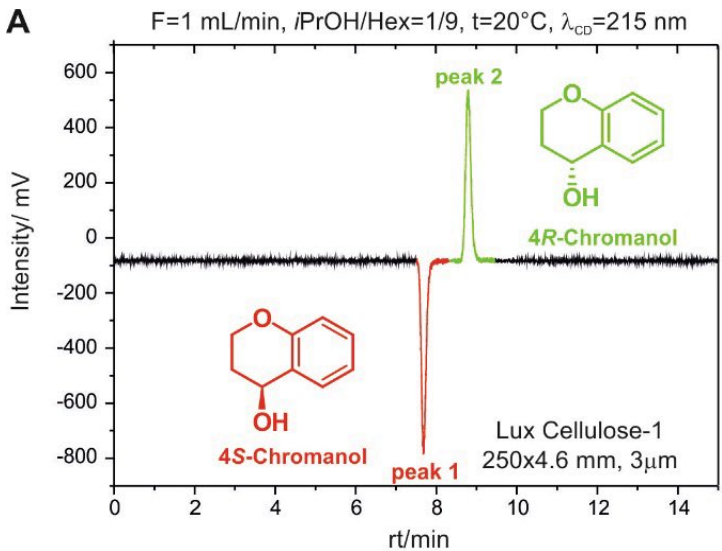

excitations: two strong positive (Ex. 2 and Ex.3) and one negative (Ex. 4) (Figure 5).

These results, combined with the earlier analysis of the optical properties of the chiral compound 3 and $4 R$-chromanol, let us assume that one of the transitions in question will occur within the amide chromophore and the two remaining within the chromanol part. However, the molecular orbital (MO) analysis of compound 2 showed that all electronic transitions within the band at around $220 \mathrm{~nm}$ (Ex.2, Ex.3, and Ex.4) are complex and both chromophores, i.e., amide and chromanol, are strongly involved. However, despite the interaction between the chromophores, excitation of the amide electrons has the greatest impact on the transition Ex. 3 at $213 \mathrm{~nm}$ and the shape of the orbital MO indicates the amide - type $n \rightarrow \pi^{*}$ transition. The relatively high value of $\mathrm{R}_{\mathrm{vel}}=+15.5 \cdot 10^{-40} \mathrm{cgs}$ for this transition determines the sign of the Cotton effect diagnostic for the helicity rule and is consistent with the rule. Thus, the tricyclic oxaanalogues of cephalosporine under study with the $6 R$ absolute configuration possess positive band at around $220 \mathrm{~nm}$ while for $6 S$ compounds the band is negative.

\section{Conclusion}

Combination of HPLC with the on-line recording of ECD spectra together with the theoretical analysis of chiroptical properties are fast,

B

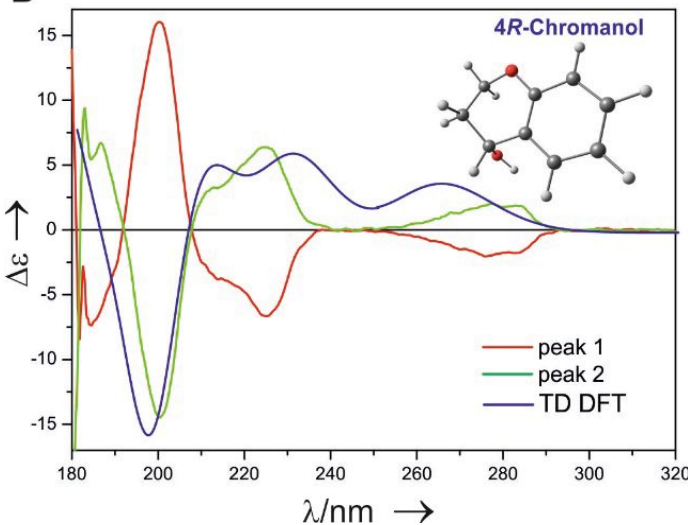

Figure 3: A) CD chromatogram of 4-chromanoles separated on Lux Cellulose-1, $3 \mu \mathrm{m}, 250 \times 4.6 \mathrm{~mm}$ column $\left(\mathrm{F}=1 \mathrm{~mL} / \mathrm{min}, \mathrm{t}=20^{\circ} \mathrm{C}, \lambda_{\mathrm{cD}}=215 \mathrm{~nm}\right.$, isocratic mode iPrOH:Hex=1:9, v:v) B) on-line recorded CD spectra of 4-chromanol enantiomers (4R - green curve, $4 S$ - red curve) compared to TD-DFT calculated ECD spectrum of $4 R$-chromanol (navy blue curve).

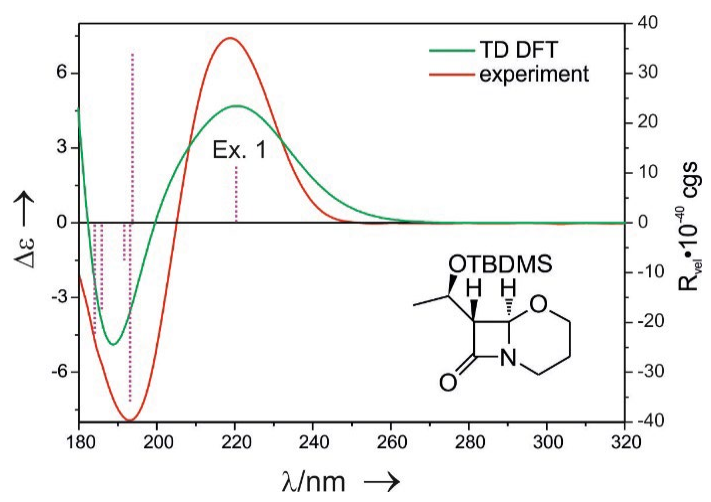

Figure 4: TD-DFT calculated ECD spectrum of compound 3 (green curve) compared to the experimental spectrum (red curve).

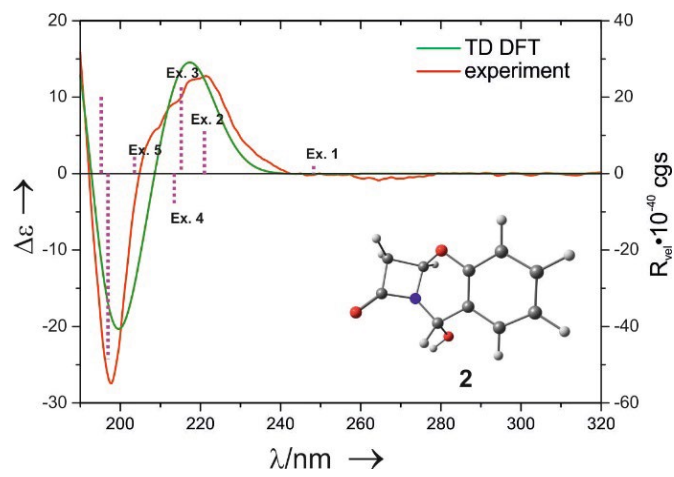

Figure 5: TD-DFT calculated ECD spectrum of compound $6 R-2$ recorded online after HPLC separation of enantiomerically enriched mixture of 2 (green curve) compared to the experimental spectrum of $6 R-2$ (red curve). 
Citation: Woźnica M, Frelek J (2016) Application of On-line HPLC-CD Detection in Separation and Structure Elucidation of Tricyclic Cephalosporine Oxaanalogues with Chromane Moiety. J Bioanal Biomed 8: e136. doi:10.4172/1948-593X.1000e136

reliable and valuable tool in structure elucidation of newly isolated or synthetized compounds with the potential biological activity.

\section{Acknowledgement}

This work was supported by the Polish Ministry of Science and Higher Education Grant luventus Plus IP2011 16771. All computational calculations were performed at the Interdisciplinary Centre for Mathematical and Computational Modeling (ICM) of University of Warsaw (grant No. G32-15).

\section{References}

1. Marquardt D, Williams JA, Kučerka N, Atkinson J, Wassall SR, et al. (2013) Tocopherol Activity Correlates with Its Location in a Membrane: A New Perspective on the Antioxidant Vitamin E. Journal of the American Chemical Society 135: 7523-7533.

2. Kamat DP, Tilve SG, Kamat VP, Kirtany JK (2015) Syntheses and Biological Activities of Chroman - 2 -ones A Review. Organic Preparations and Procedures International 47: 1-79.

3. Emami S, Ghanbarimasir Z (2015) Recent advances of chroman - 4 - one derivatives: Synthetic approaches and bioactivities. European Journal of Medicinal Chemistry 93: 539-563.

4. Cabaret D, Adediran SA, Garcia Gonzalez MJ, Pratt RF, Wakselman M
(1999) Synthesis and Reactivity with $\beta$ - Lactamases of "Penicillin - like" Cyclic Depsipeptides. The Journal of Organic Chemistry 64: 713-720.

5. Essack SY (2001) The Development of $\beta$ - Lactam Antibiotics in Response to the Evolution of $\beta$-Lactamases. Pharmaceutical Research 18: 1391-1399.

6. http://www.ncbi.nlm.nih.gov/books/NBK207662/.

7. http://www.arkat-usa.org/get-file/49636/.

8. Kozioł A, Furman BO, Frelek J, Woźnica M, Altieri E, et al. (2009) Chira Base Catalyzed Enantioselective Synthesis of 4 - Aryloxyazetidinones and 3 4 - Benzo - 5 - oxacephams. The Journal of Organic Chemistry 74: 5687-5690.

9. Frelek J, Kowalska P, Masnyk M, Kazimierski A, Korda A et al. (2007) Circular Dichroism and Conformational Dynamics of Cephams and Their Carba and Oxa Analogues. Chemistry A European Journal 13: 6732-6744.

10. Woźnica M, Kowalska P, Łysek R, Masnyk M, Gorecki M, et al. (2010) Stereochemical Assingment of $\beta$-lactam Antibiotics and their Analogues by Electronic Circular Dichroism Spectroscopy. Current Organic Chemistry 14: 1022-1036.

11. Woźnica M, Masnyk M, Stecko S, Mames A, Furman B, et al. (2010) Structure - Chiroptical Properties Relationship of Carbapenams by Experiment and Theory. The Journal of Organic Chemistry 75: 7219-7226.

12. Frisch MJ, Trucks GW, Schlegel HB, Scuseria GE, Robb Maski VG, etal. (2009) Official Gaussian 09 Literature Citation Wallingford CT USA. 